\title{
'Smart' intravenous pumps: how smart are they?
}

\section{Bryony Dean Franklin ${ }^{1,2}$}

\begin{abstract}
${ }^{1}$ Centre for Medication Safety and Service Quality, Imperial College Healthcare NHS Trust/ UCL School of Pharmacy, London, UK

${ }^{2}$ Department of Practice and Policy, UCL School of Pharmacy, London, UK
\end{abstract}

\section{Correspondence to} Professor Bryony Dean Franklin, Pharmacy Department, Charing Cross Hospital, Imperial College Healthcare NHS Trust/UCL School of Pharmacy, London W6 8RF, UK; bryony.deanfranklin@ imperial.nhs.uk

Accepted 17 March 2016 Published Online First

7 April 2016

\section{SLinked}

- http://dx.doi.org/10.1136/ bmjqs-2015-004465

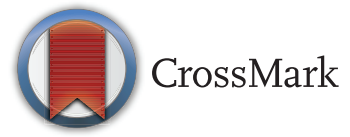

To cite: Franklin BD. BMJ Qual Saf 2017;26:93-94.
Smart pumps, incorporating dose error reduction software, are widely promoted as a patient safety intervention. ${ }^{12}$ This software checks programmed intravenous infusion rates against preset limits for each drug in the pump's 'drug library' with the aim of reducing the risk of infusion rates that are too high or too low. Smart pumps were reported to be in use in $68 \%$ of US hospitals in $2011,^{3}$ although this figure does not tell about how they are used nor in which clinical areas. A UK study indicated less widespread use and that although smart pumps may be in use within an organisation, they may only be used in some clinical areas or for certain kinds of infusion. ${ }^{4}$

Although widely advocated, as with many other patient safety interventions, ${ }^{5-7}$ the evidence for smart pumps' benefit is not clear-cut. No conclusive evidence shows that smart pumps do indeed prevent medication errors and adverse drug events, and we know little about the kinds of errors that still occur with their use. $^{89}$

In this issue, Schnock et $a l^{10}$ aim to shed light on some of these issues by documenting the prevalence and types of errors associated with intravenous infusions in 10 US hospitals using smart pumps. Pairs of observers visited participating clinical areas and identified discrepancies between each infusion and its corresponding medication order plus relevant organisational policies. This method can reveal only errors visually apparent from an infusion in progress. Any errors in the preparation of the infusion, such as the wrong concentration being prepared, will not be identified unless the corresponding label is also incorrect. This approach also focuses on errors in medication administration and does not include prescribing errors involving incorrect infusion rates, which smart pumps may also have a role in preventing. Even so, the paper suggests a very high error rate, with $60 \%$ of 1164 observed infusions reported as having one or more errors. At face value, this figure seems very high, but it includes procedural violations as well as what would usually be considered to be medication administration errors and, as the authors point out, very few (5 of the 1691 errors identified in 1164 infusions) were judged to be potentially harmful.

The five potentially significant medication administration errors comprised four 'errors that would have required increased monitoring to preclude harm' (category D) and one 'error likely to cause temporary harm' (category E). The four category $D$ errors consisted of two wrong rate errors, one omission error and one expired drug error; the category $\mathrm{E}$ error was an omission error. The error rate based on these five more serious errors is just $0.4 \%$ of infusions-more than a hundred times lower than the headline figure of $60 \%$-perhaps suggesting that intravenous infusions are relatively safe.

This 100-fold discrepancy highlights two issues. The first is the extent to which procedural violations are important. On the one hand, procedural violations may be a useful indicator of the underlying culture and may affect whether or not patients feel that their care is safe. One study showed an association between a particular procedural violation (not checking patient identification) and medication administration errors, with the authors suggesting that not checking patient identity may indicate a general failure to follow administration protocols. ${ }^{11}$ On the other hand, including minor procedural failures that do not result in medication administration errors may detract from the more important issues and risk creating the perception of the research being less relevant to practice. For example, Schnock et al included procedural issues such as discontinued infusions being disconnected from the patient but still connected to the pump, which are unlikely to be considered 
important by most healthcare professionals. Procedural violations may also reflect policies that are no longer fit for purpose. Schnock et $a l^{10}$ highlight that some policies required the actual start time of infusions to be documented on the infusion label, but that this information will be captured automatically in organisations using barcode medication administration systems and electronic medication administration records. In this context, adding this information by hand to the label can be viewed as a redundant step.

The second issue is the challenge of interpreting the literature in this field: different researchers and practitioners may have different views on what should count as errors. This is a particular issue for quantitative studies of medication administration errors, making it difficult to compare different studies even where the same data collection methods are used. ${ }^{12}$

Schnock et al also reveal important differences among the 10 participating hospitals. There was wide variation in non-adherence to smart pump use (ranging from $0 \%$ to $38 \%$ of infusions for which the smart pump was not used at all or the drug library bypassed, negating any benefits of dose range checking) and other types of medication administration error (ranging from 6\% to $60 \%$ infusions, excluding those relating to smart pump non-adherence). This 10 -fold difference in medication administration error rates is largely accounted for by variation in the prevalence of medications being administered without an accompanying medication order, mainly the infusion of fluids at a low rate to keep the vein open, which ranged from $3 \%$ to $53 \%$ of observed infusions across the 10 sites. As well as events considered to be medication administration errors, there was also wide variation in adherence to other procedural issues. Policy violations were defined based on the policy in place at each organisation and the prevalence and types of violation will therefore depend on the policy. For example, if a hospital policy stipulates a large number of requirements for the information on an infusion bag label, there are more opportunities for violation of these requirements than in a hospital that requires only the patient's name and identification number. Perhaps importantly, the 10 study hospitals were also a convenience sample, selected from attendees at a healthcare technology safety meeting who volunteered to participate. Infusion practices may be even more diverse beyond this self-selected sample.

In relation to the role of smart pumps, it seems that the picture remains unclear. Serious errors occurred relatively rarely in these 10 hospitals using smart pumps, but procedural violations were common. Smart pumps are likely to be only as smart as the rest of the system in which they operate. As with many healthcare technologies, their benefits are likely to depend on how they are used, how they are integrated within practice and the interface between humans and technology. Adequately exploring these issues will likely require qualitative as well as quantitative methods. ${ }^{13}$ Even if used as part of a closed loop system, integrated with computerised prescriber order entry and barcode medication administration systems, smart pumps are unlikely to affect adherence to other procedures relating to the safe administration of intravenous infusions. It is important that we do not regard smart pumps as a 'plug and play' technology to be added into existing systems for intravenous medication administration; instead they should be used as an opportunity for wider transformation of the whole system.

Funding The Centre for Medication Safety and Service Quality is partly funded by the National Institute for Health Research (NIHR) Imperial Patient Safety Translational Research Centre. The views expressed are those of the author(s) and not necessarily those of the NHS, the NIHR or the Department of Health.

Competing interests None declared.

Provenance and peer review Commissioned; internally peer reviewed.

\section{REFERENCES}

1 Institute of Medicine. To err is human: building a safer health system. Report of the Committee on Quality of Health Care in America, Institute of Medicine. Washington: National Academy Press, 2000.

2 Department of Health. An organisation with a memory. London: Stationery Office, 2000.

3 Pedersen CA, Schneider PJ, Scheckelhoff DJ. ASHP national survey of pharmacy practice in hospital settings: dispensing and administration-2011. Am J Health Syst Pharm 2012;69:768-85.

4 Iacovides I, Blandford A, Cox A, et al. Infusion device standardisation and dose error reduction software. Br J Nurs 2014;23:S16-24.

5 Raban MZ, Westbrook JI. Are interventions to reduce interruptions and errors during medication administration effective?: A systematic review. BMJ Qual Saf 2014;23:414-21.

6 Catchpole K, Russ S. The problem with checklists. BMJ Qual Saf 2015;24:545-9.

7 Shojania KG, Thomas EJ. Trends in adverse events over time: why are we not improving? BMJ Qual Saf 2013;22:273-7.

8 Ohashi K, Dalleur O, Dykes PC, et al. Benefits and risks of using smart pumps to reduce medication error rates: a systematic review. Drug Saf 2014;37:1011-20.

9 Hertzel C, Sousa VD. The use of smart pumps for preventing medication errors. J Infus Nurs 2009;32:257-67.

10 Schnock et al. The frequency of intravenous medication administration errors related to smart infusion pumps: a multi hospital observational study. BMJ Qual Saf 2017;26:131-40

11 Westbrook JI, Rob MI, Woods A, et al. Errors in the administration of intravenous medications in hospital and the role of correct procedures and nurse experience. BMJ Qual Saf 2011;20:1027-34.

12 McLeod MC, Barber N, Franklin BD. Methodological variations and their effects on reported medication administration error rates. BMJ Qual Saf 2013;22:278-89.

13 Blandford A, Furniss D, Lyons I, et al. Exploring the Current Landscape of Intravenous Infusion Practices and Errors (ECLIPSE): protocol for a mixed methods observational study. BMJ Open 2016;6:e009777. 